\title{
Research Paper: Comparative Analysis of Platelet-rich Plasma Treatment for Chronic Synovitis in Patients With Hemophilia: A Six-month Follow-up
}

\author{
1. Department of Orthopaedic and Traumatology, General Hospital Juan A Fernández, Cerviño, CABA, Argentina. \\ 2. Haemophilia Foundation, Soler, CABA, Argentina.
}

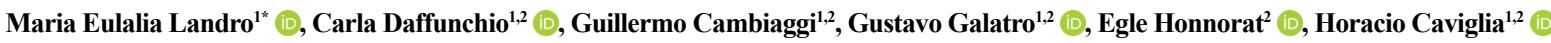

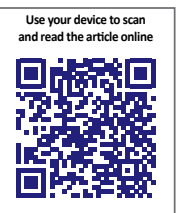

Citation Landro ME, Daffunchio C, Cambiaggi G, Galatro G, Honnorat E, Caviglia H. Comparative Analysis of Plateletrich Plasma Treatment for Chronic Synovitis in Patients With Hemophilia: A Six-month Follow-up. Journal of Research in Orthopedic Science. 2021; 8(3):163-170. http://dx.doi.org/10.32598/JROSJ.8.3.787.1

doi http://dx.doi.org/10.32598/JROSJ.8.3.787.1

Keywords:

Inhibitor, Musculoskeletal, Hemophilia, Platelet-rich Plasma, Synovitis

\begin{abstract}
A B S T RA C T
Background: Inhibitor development is the main treatment-related complication in patients with hemophilia. Degenerative joint lesions caused by repeated bleeding episodes are more common in patients with inhibitors than in those without inhibitors. We introduced Platelet-rich Plasma (PRP) treatment to reduce joint damage and provide symptomatic relief.

Objectives: The aim of this study was to compare PRP treatment outcomes in two groups of patients with hemophilia presenting synovitis, with or without inhibitors, at 3- and 6-month follow-ups.

Methods: We treated 87 patients with 143 affected joints divided into two groups: 78 patients without inhibitors with 129 affected joints and a mean age of 25.8 years, and 9 patients with inhibitors with 14 affected joints and a mean age of 16.3 years.

Results: The difference in mean age between the two groups was statistically significant $(\mathrm{P}=0.007)$. The number of bleeding episodes, Visual Analogue Scale (VAS), and Hemophilia Joint Health Score (HJHS) before treatment and after three and six months showed no statistically significant differences between groups. We demonstrated that PRP treatment decreased the number of bleeding episodes, pain levels, and HJHS in the whole study population $(\mathrm{P}<0.001)$ No differences were found between patients with and without inhibitors.

Conclusion: PRP was proved to be effective for synovitis treatment in patients with hemophilia with and without inhibitors.
\end{abstract}

\footnotetext{
* Corresponding Author:

María Eulalia Landro, MD.

Address: Department of Orthopaedic and Traumatology, General Hospital Juan A Fernández, Cerviño 3356, 1425, CABA, Argentina.

Phone: +54 (911) 48082600

E-mail: mlandro@gmail.com
} 


\section{Introduction}

H emophilia is a serious inherited bleeding disorder resulting from deficiency of coagulation factor VIII (hemophilia A) or coagulation factor IX (hemophilia B). Effective therapies have arisen to replace the missing factor and restore normal coagulation, but they can lead to the development of neutralizing anti-drug antibodies (inhibitors) [1].

Inhibitor development is the main complication in the treatment of Patients With Hemophilia (PWH). Approximately 25 to $30 \%$ of severe hemophilia A patients and 3 to $5 \%$ of hemophilia $\mathrm{B}$ patients under prophylactic treatment develop inhibitors [2].

An additional complication of the pharmacological approach is that alternative hemostatic agents are required, and these are not as safe or efficient as factor concentrates [3]. Inhibitors are also associated with significant morbidity, mortality, disability, bleeding complications, and increased health care costs [3-5].

Due to the difficulty in treating bleeding episodes in the musculoskeletal system of patients with inhibitors, degenerative joint lesions are more common in these patients than in patients without inhibitors $[4,6]$. The management of patients with inhibitors remains a significant challenge for multidisciplinary teams treating PWH.

We introduced Platelet-rich Plasma (PRP) treatment for chronic synovitis in PWH two years ago, to reduce joint damage caused by bleeding and provide symptomatic relief [7-9]. This treatment relies on the biological properties of PRP, and the capacity of growth factors and proteins released by platelets to reduce clinical symptoms and enhance healing mechanisms and tissue regeneration [10-12].

\section{Objectives}

The aim of this study was to compare PRP treatment outcomes in two groups of patients with hemophilia presenting synovitis, with or without inhibitors, at 3- and 6-month follow-ups.

\section{Methods}

We evaluated patients with chronic synovitis (diagnosed by clinical exam, Rx, ultrasound and/or MRI) before intervention and six months after PRP injection.
Patients with grade V arthropathy (osseous ankylosis), according to Arnold and Hilgartner's radiological classification and clinical exam, and those with skin defects or active joint infection were also excluded.

We divided this population into two groups: patients with inhibitors and patients without inhibitors. Parameters evaluated were age, hemophilia type, number of Bleeding Episodes (BE), Visual Analogue Scale (VAS), Hemophilia Joint Health Score (HJHS) for each affected joint, and the number of affected joints in each patient.

The study population included 87 patients with 143 affected joints. Incidence was 1.64 (1.37-1.91) affected joints per patient.

The mean age of the whole population was $24.8 \pm 10.1$ years. Eighty-three patients $(95.4 \%)$ had hemophilia A and four patients $(4.6 \%)$ had hemophilia B. Seventy-seven patients $(88.5 \%)$ presented severe hemophilia and ten patients $(11.5 \%)$ presented moderate hemophilia.

The group of patients without inhibitors included 78 patients $(89.7 \%)$ with 129 affected joints. Incidence was 1.65 (1.36-1.94) affected joints per patient and mean age was $25.8 \pm 9.9$ years. Seventy-four patients $(94.9 \%)$ had hemophilia A and four patients (5.1\%) had hemophilia B. Sixty-eight patients (87.2\%) had severe hemophilia and ten patients $(12.8 \%)$ had moderate hemophilia.

The group of patients with inhibitors included nine patients $(10.3 \%)$ with 14 affected joints. The incidence was $1.56(0.85-2.61)$ affected joints per patient. The mean age was $16.3 \pm 7.3$ years. All patients with inhibitors had severe hemophilia A. Table 1 shows a description of the study population.

A total of 143 joints were treated: 66 knees (46.1\%), 30 elbows (21\%), 46 ankles (32.2\%), and one shoulder $(0.7 \%) .46$ patients $(52.9 \%)$ had one affected joint, 41 patients $(47.1 \%)$ had more than one affected joint, 27 patients $(31 \%)$ had two affected joints, 13 patients $(15.0 \%)$ had three affected joints, and one patient $(1.1 \%)$ had four affected joints.

In the group of patients without inhibitors, 129 joints were treated: 60 knees (46.5\%), 26 elbows (20.1\%), 42 ankles $(32.6 \%)$, and one shoulder (0.8\%). 40 patients $(51.3 \%)$ had one affected joint, 38 patients $(48.7 \%)$ had more than one affected joint, 26 patients $(33.3 \%)$ had two affected joints, 11 patients $(14.1 \%)$ had three affected joints, and one patient $(1.3 \%)$ had four affected joints. 
Among patients with inhibitors, 14 joints were treated: 6 knees (42.8\%), 4 elbows (28.6\%), and 4 ankles (28.6\%). Six patients $(66.7 \%)$ had one affected joint, one patient $(11.1 \%)$ had two simultaneously affected joints, and two patients $(22.2 \%)$ had three affected joints (two of them: simultaneous).

We noticed that the presence of synovitis in patients with inhibitors tended to affect different joints simultaneously. Before PRP treatment, patients with inhibitors were studied to measure inhibitor titer, followed by the indication of the bypassing scheme. In patients without inhibitors, the inhibitor test was performed as well, for negative confirmation $(<0.6 \mathrm{BU} / \mathrm{ml})$.

The affected joints were treated with fresh PRP. In brief, PRP was prepared as follows: blood was extracted with the BD vacutainer ${ }^{\circledR}$ (USA) collection set (butterfly needle, holder, and $8.5 \mathrm{ml}$ ACD tubes). We used a single-speed method for PRP preparation consisting of 8-minute centrifugation at $1800 \mathrm{rpm}$ or $360 \mathrm{~g}$ (Presvac, Buenos Aires, Argentina) at room temperature. Then, the PRP fraction was carefully harvested with a pipette (in order to minimize white or red blood cell aspiration) and placed in a sterile syringe. This procedure was performed in a Class II biosafety cabinet (Biobase, Shandong, China). The upper half of the plasma (PPP: Platelet-poor plasma) and the rest of the blood were discarded. Also, $1 \mathrm{ml}$ of the PRP was reserved for platelet count in a Coulter LH 750 Analyser (Beckman Coulter, Inc., Indiana, USA).

After blood extraction, patients were given the corresponding coagulation factors indicated by the treating hematologist. The recommendation was to infuse factor VIII or IX, as appropriate, 10 minutes before PRP injection, to reach a clotting factor activity of $50 \%$ and to maintain factor levels $\geq 30 \%$ for the next three days.

Patients with inhibitors received one dose of 150-200 $\mu \mathrm{g} / \mathrm{kg}$ recombinant activated Factor VII (rFVIIa), followed by three doses of $90 \mu \mathrm{g} / \mathrm{kg}$, every three hours. A dose of $200-300 \mu \mathrm{g} / \mathrm{kg}$ was administered daily over the next three days. Intra-articular PRP injections were performed under sterile conditions in the operating room. The average delay between blood extraction and PRP injection was one hour.

\section{Study design and statistical analysis}

We performed a quasi-experimental study to analyze the variables (BE, VAS, and HJHS), before and after PRP intra-articular injection during a 6-month follow-up.
Continuous variables presenting normal distribution were expressed as mean and Standard Deviation (SD). Otherwise, the median and Interquartile Range (IQR) were reported. To determine distribution symmetry, the Shapiro-Wilk or Kolmogorov-Smirnov tests were used, as appropriate. Categorical variables were reported as absolute numbers and percentages. For categorical variables, Chi-square or Fisher's exact tests were used. For continuous variables, Student's t-test or Mann-Whitney test were performed.

To evaluate changes in the number of bleeding episodes, VAS and HJSH score before treatment and three and six months after injection, an analysis of variance of repeated measures was performed. If normality of the transformed data was not assumed, Friedman's nonparametric test, Wilcoxon test, and Bonferroni test were used for post hoc comparisons. In addition, differences between groups were assessed by the non-parametric Analysis of Covariance (ANCOVA) alternative, the Quade test [13]. A p-value below 0.05 was considered significant. Data obtained three and six months after treatment were independently analyzed. All statistical analyses were performed using SPSS Statistics v. 24 software (SPSS Inc., Chicago, IL).

\section{Results}

The mean age of the total population was 24.8 years. The mean age of patients without inhibitors was 25.8 years, while the mean age of patients with inhibitors was16.3 years. The difference in the mean age between both groups was statistically significant $(\mathrm{P}=0.007)$.

The incidence of affected joints in the study population was 1.64 joints per patient. In patients without inhibitors, it was 1.65 , and in patients with inhibitors, it was 1.56. The difference between groups was not statically significant $(\mathrm{P}=0.86)$.

The mean volume of extracted blood for PRP preparation was $15 \mathrm{ml}$ (12-24 $\mathrm{ml})$. This volume depended on the number of joints to be treated simultaneously. The mean volume of injected PRP was $4 \mathrm{ml}(3-7 \mathrm{ml})$ for each affected joint and varied according to the size of the joint treated. Mean platelet count was $363 \times 10^{3}$ platelets $/ \mathrm{ml}$ $\left(260-580 \times 10^{3}\right.$ platelets $\left./ \mathrm{ml}\right)$. The mean platelet concentration factor in PRP was 2. There were no complications either during blood extraction or intra-articular PRP injection in any patient.

All patients were evaluated for the number of BE, VAS, and HJHS for each affected joint before treatment and at 
Table 1. Description of the study population

\begin{tabular}{|c|c|c|c|c|}
\hline \multicolumn{2}{|c|}{ Parameters } & \multicolumn{3}{|c|}{ Mean $\pm S D / N o . ~(\%)$} \\
\hline & & Total $(n=87)$ & With Inhibitor $(n=9)$ & Without Inhibitor $(n=78)$ \\
\hline & & $24.8 \pm 10.1$ & $16.3 \pm 7.3$ & $25.8 \pm 9.9$ \\
\hline \multirow{3}{*}{ Hemophilia type } & A & 83(95.4) & $9(100)$ & 74(94.9) \\
\hline & & & & \\
\hline & B & $4(4.6)$ & $0(0)$ & $4(5.1)$ \\
\hline \multirow{3}{*}{ Hemophilia severity } & Moderate & 10(11.4) & $0(0)$ & 10(12.8) \\
\hline & & & & \\
\hline & Severe & $77(88.6)$ & $9(100)$ & 68(87.2) \\
\hline
\end{tabular}

3 and 6 months follow-up. Table 2 shows data analysis of the whole population before treatment (baseline) and three and six months post-intervention.

Changes in BE, VAS, and HJHS between the baseline and 3-month follow-up, and between baseline and 6-month follow-up were statistically significant $(\mathrm{P}<0.001)$ for both groups of patients and did not vary significantly three and six months after treatment. No statistically significant differences in BE, VAS, and HJSH were observed between groups at 3- and 6-month follow-up (Tables 3 and 4).

Regarding BE, all patients with inhibitors had at least one BE before treatment. Only a few patients without inhibitors had no BE before treatment. After treatment, the frequency of $\mathrm{BE}$ decreased in both groups. In patients with inhibitors, the decrease in the number of BE persisted over six months of follow-up.

The median VAS and HJHS values were different between both groups of patients. VAS values of both groups decreased at 3- and 6-month follow-ups. At 6-month follow-up, both groups presented similar VAS values.

Baseline HJHS values were similar between both groups. Three months after treatment, HJHS decreased in both groups. Six months after treatment, HJHS remained unaltered in patients with inhibitors and slightly decreased in patients without inhibitors. These differences were not statistically significant.

\section{Discussion}

Considering that $80 \%$ of bleeding episodes occur in the musculoskeletal system, the management of hemophilia requires a multidisciplinary team, including hematologists, orthopedic surgeons, rehabilitation physicians, physiotherapists, and biologists [14]. Unfortunately, the vast majority of the world's hemophilic population does not receive appropriate hematological treatment [15].

There is a higher proportion of patients with inhibitors among severe hemophilia A patients than among patients with moderate or mild hemophilia A (25-40\% vs. 5-15\%) [4]. In patients with hemophilia B, the risk of inhibitor development is about $1-5 \%$, usually occurring in patients with severe hemophilia B [16].

In our study population, all patients with inhibitors had severe hemophilia A, while patients without inhibitors presented severe or moderate hemophilia A and B. The patients with inhibitors were younger than patients with-

Table 2. Statistical analysis of total study population

\begin{tabular}{ccccc}
\hline Parameters & Basal & Three Months & Six Months \\
\hline BE & $2(1-3)$ & $0(0-1)$ & $0(0-1)$ & $1(0-4)$ \\
VAS & $5(3-7)$ & $2(0-4)$ & $11(9-13)$ & $<0.001$ \\
HJSH & $14(11.5-17)$ & $12(9-14)$ & $<0.001$ & Orthopedic Science
\end{tabular}

BE: Bleeding Episodes; HJSH: Hemophilia Joint Health Score; VAS: Visual Analogue Scale; Basal: before treatment. Data are presented as median and range; Test: Friedman and Bonferroni tests 
Table 3. Group Analysis at three months follow-up

\begin{tabular}{ccccc}
\hline Parameters & Groups & Basal & Three Months & P \\
\hline \multirow{2}{*}{ BE } & With inhibitor & $2(1-3)$ & $0(0-1)$ & 0.204 \\
& Without inhibitor & $2(1-3)$ & $0(0-1)$ & 0.686 \\
VAS & With inhibitor & $2.5(1-6.2)$ & $1(0-2.5)$ & $2(0-4)$ \\
& Without inhibitor & $5(3-7)$ & $12(9-14)$ & 0.737 \\
\hline
\end{tabular}

Orthopedic Science BE: Bleeding Episodes; HJSH: Haemophilia Joint Health Score; VAS: Visual Analogue Scale; Basal: Before treatment. Data are presented as median and range; Test: ANCOVA

out inhibitors (16.3 vs. 25.8 years), and these differences were statistically significant $(\mathrm{P}=0.007)$.

Forsyth et al. reported that joint damage evolves faster in patients with inhibitors $[17,18]$. This difference is probably due to two factors. In Argentina, most patients under 18 years without inhibitors receive prophylaxis, which prevents synovitis. In addition, patients with inhibitors are treated as soon as possible after the onset of clinical symptoms [3]. Hematologists and traumatologists are often reluctant to treat inhibitor patients for fear of complications and delay treatment of synovitis. Primary prophylaxis is the standard care for patients with severe hemophilia without inhibitors, to prevent bleeding and deterioration of the joint and improve patients' quality of life [19].

The incidence of affected joints in our total study populations was 1.64 per patient. In patients without inhibitors, it was 1.65 , and in patients with inhibitors, it was 1.56 per patient. The difference between groups was not statically significant $(\mathrm{P}=0.86)$. In patients without inhibitors, the most frequently affected joint was the knee $(46.5 \%)$, followed by the ankle $(35.6 \%)$, the elbow $(20.1 \%)$, and the shoulder $(0.8 \%)$. In patients with inhibitors, the most frequently affected joint was also the knee $(42.8 \%)$, but the ankle and the elbow were equally affected $(28.6 \%)$. We observed that patients with inhibitors tended to develop synovitis in more than one joint simultaneously more frequently than patients without inhibitors.

Intra-articular PRP can be administered in patients with inhibitors without any complications and as successfully as in patients without inhibitors [8,9].

We found no association between platelet count and clinical outcome in any of the groups studied.

This study demonstrated the benefits of PRP treatment for chronic synovitis, as it decreased the number of bleeding episodes, pain levels, and HJHS in our total

Table 4. Group analysis at six months follow-up

\begin{tabular}{|c|c|c|c|c|}
\hline Parameter & Groups & Basal & Six Months & $\mathbf{P}$ \\
\hline \multirow{3}{*}{ BE } & With inhibitor & $2(1-3)$ & $0(0-1)$ & \\
\hline & & & & 0.515 \\
\hline & Without inhibitor & $2(1-3)$ & $0(0-1)$ & \\
\hline \multirow{3}{*}{ VAS } & With inhibitor & $2(1-7)$ & $1(0-5)$ & \\
\hline & & & & 0.367 \\
\hline & Without inhibitor & $5.5(3-7)$ & $1(0-4)$ & \\
\hline \multirow{3}{*}{ HJHS } & With inhibitor & $14(12-16)$ & $12(9-15)$ & \\
\hline & & & & 0.643 \\
\hline & Without inhibitor & $14(11-17)$ & $11(8-13)$ & \\
\hline
\end{tabular}

Orthopedic Science

BE: Bleeding Episodes; HJSH: Haemophilia Joint Health Score; VAS: Visual Analogue Scale; Basal: Before treatment. Data are presented as median and range; Test: ANCOVA and Quade test 
study population. These results, before and after treatment, were statistically significant $(\mathrm{P}<0.001)$. Both groups analyzed benefited from PRP treatment without statistically significant differences between them. This suggests that PRP may be equally effective for patients with and without inhibitors.

There were no additional complications when administering PRP to patients with inhibitors. We recommend immediate treatment of joints at risk of damage, target joints, and synovitis in inhibitor patients. In addition to PRP treatment, patients with inhibitors should receive four weeks of prophylaxis to prevent additional bleeding. The synovial response should be evaluated with ultrasound imaging to evaluate response to treatment.

Hemarthrosis is the hallmark of hemophilia. Several reports suggest that two hemarthrosis episodes occurring in the same joint may result in permanent joint damage $[18,20]$. Proper management and monitoring of patients with inhibitors are extremely challenging. Novel alternative therapies to provide better outcomes in these patients will be developed in the future [14].

PRP is a minimally invasive, safe, low-cost treatment that can be used in PWH, including those with inhibitors. It requires only the patient's own blood, a centrifuge, and a qualified doctor to perform the joint puncture. This procedure is simple enough for PRP treatment to be used globally. It can be reapplied several times if required with no adverse effects since it is obtained from the patient's own blood [21].

The present study has some limitations, due to the small number of patients with inhibitors and the quasiexperimental study design. We must consider that we studied a small population of PWH who had synovitis and also those who had inhibitors.

\section{Conclusion}

PRP treatment reduced the symptoms of chronic synovitis in patients with hemophilia with and without inhibitors, in the absence of complications and improved the quality of life of patients with hemophilia. According to our results, we propound that PRP could help prevent joint bleeding episodes and pain, reducing the risk of joint damage in PWH with and without inhibitors. Further research is needed to better understand the mechanism of action of PRP treatment in these patients.

\section{Ethical Considerations}

\section{Compliance with ethical guidelines}

This study was approved by the Institutional Ethics Committee (Ref. protocol: 201652/2017) according to law 3301, decree 58/2011, resolution 485/MSGC/2011, Buenos Aires City Government. This study followed the principles of the Declaration of Helsinki. Informed consent was obtained from each patient.

\section{Funding}

This research did not receive any grant from funding agencies in the public, commercial, or non-profit sectors.

\section{Authors' contributions}

Conceptualization and Supervision: Dr. Horacio Caviglia; Methodology, Investigation, Writing - editing original draft: Maria E. Landro; Writing - review: Carla Daffunchio, Egle Honnorat, Gustavo Galatro, and Guillermo Cambiaggi.

\section{Conflict of interest}

The authors declared no conflict of interests.

\section{References}

[1] Young G. How I treat children with hemophilia and inhibitors. Br J Haematol. 2019; 186(3):400-8. [DOI:10.1111/ bjh.15942]

[2] Atilla B, Güney-Deniz H. Musculoskeletal treatment in hemophilia. EFORT Open Rev. 2019; 4(6):230-9. [DOI:10.1302/2058-5241.4.180068]

[3] Rocino A, Franchini M, Coppola A. Treatment and prevention of bleeds in hemophilia patients with inhibitors to factor VIII/IX. J Clin Med. 2017; 6(4):46. [DOI:10.3390/jcm6040046]

[4] Santagostino E, Young G, Escuriola C, Ettingshausen V, Jimenez-Yuste, Carcao M. Inhibitors: A need for eradication? Acta Haematol. 2019; 141(3):151-5. [DOI:10.1159/000495454]

[5] Morfini M, Haya S, Tagariello G, Pollmann H, Quintana M, Siegmund B, et al. European study on orthopaedic status of hemophilia patients with inhibitors. Hemophilia. 2007; 13(5):606-12. [DOI:10.1111/j.1365-2516.2007.01518.x]

[6] Morfini M. Articular status of hemophilia patients with inhibitors. Hemophilia. 2008; 14(14 S 6):20-2. [DOI:10.1111/ j.1365-2516.2008.01885.x] 
[7] Teyssler P, Kolostova K, Bobek V. The impact of platelet-rich plasma on chronic synovitis in hemophilia. Acta Orthop Belg. 2014; 80(1):11-7. [PMID]

[8] Caviglia H, Landro ME, Daffunchio C, Galatro G, Douglas Price AL, Salgado P, et al. Platelet rich plasma for chronic synovitis treatment in patients with hemophilia. Hemophilia. 2017; 23(4):613-9. [DOI:10.1111/hae.13212]

[9] Landro ME, Daffunchio C, Cambiaggi G, Galatro G, Salgado $\mathrm{P}$, Honorat E, et al. Platelet rich plasma intra articular injection for chronic synovitis treatment in patients with hemophilia one year follow up. Biomed J Sci Tech Res. 2019; 12(5). [DOI:10.26717/BJSTR.2019.12.002319]

[10] Beitzel K, McCarthy MB, Russell RP, Apostolakos J, Cote MP, Mazzocca AD. Learning about PRP using cell-based models. Muscles Ligaments Tendons J. 2014; 4(1):38-45. [PMCID]

[11] Marmotti A, Rossi R, Castoldi F, Roveda E, Michielon G, Peretti GM. PRP and articular cartilage: A clinical update. Biomed Res Int. 2015; 2015:542502. [DOI:10.1155/2015/542502]

[12] Abolfazl B, Jabalameli M, Jahansouz A, Yahyazadeh H, Heris HK, Khezri M, et al. Platelet-rich plasma injection for symptomatic knee osteoarthritis. J Res Orthop Sci. 2015; 2(3):e2022. [DOI:10.17795/soj-2022]

[13] Quade D. Rank analysis of covariance. J Amer Statist Assoc. 1967; 62(320):1187-200. [DOI:10.1080/01621459.1967.10 500925]

[14] Cuesta Barriuso R, Trelles-Martínez RO. Manual therapy in the treatment of patients with hemophilia $\mathrm{B}$ and inhibitor. BMC Musculoskelet Disord. 2018; 19(1):26. [DOI:10.1186/ s12891-018-1934-9]

[15] Mahlangu J, Oldenburg J, Callaghan M, Shima M, Santagostino E, Moore M, et al. Bleeding events and safety outcomes in persons with hemophilia A with inhibitors: A prospective, multi-centre, non-interventional study. Hemophilia. 2018; 24(6):921-9. [DOI:10.1111/hae.13612]

[16] Carcao M, Goudemand J. Inhibitors in hemophilia: A primer. Treat Hemophilia. 2018; 7. [DOI:10.1016/B978-0323-35762-3.00135-9]

[17] Forsyth AL, Rivard GÉ, Valentino LA, Zourikian N, Hoffman M, Monahan PE, et al. Consequences of intra-articular bleeding in hemophilia: Science to clinical practice and beyond. Hemophilia. 2012; 18(S 4):112-9. [DOI:10.1111/j.13652516.2012.02835.x]

[18] Gringeri A, Ewenstein B, Reininger A. The burden of bleeding in hemophilia: Is one bleed too many? Hemophilia. 2014; 20(4):459-63. [DOI:10.1111/hae.12375]

[19] Srivastava A, Brewer AK, Mauser-Bunschoten EP, Key NS, Kitchen S, Llinas A, et al. Guidelines for the management of hemophilia. Haemophilia. 2013; 19(1):e1-47. [DOI:10.1111/ j.1365-2516.2012.02909.x]

[20] van Meegeren ME, Roosendaal G, Jansen NW, Lafeber FP, Mastbergen SC. Blood-induced joint damage: The devastating effects of acute joint bleeds versus micro-bleeds. Cartilage. 2013; 4(4):313-20. [DOI:10.1177/1947603513497569]

[21] Andia I, Marffulli N. A contemporary view of platelet rich plasma therapies: Moving toward refined clinical proto- cols and precise indications. Regen Med. 2018; 13(6):717-28. [DOI:10.2217/rme-2018-0042] 
This Page Intentionally Left Blank 